MATHEMATICS OF COMPUTATION

Volume 69, Number 232, Pages 1341-1354

S 0025-5718(99)01164-3

Article electronically published on May 20, 1999

\title{
AN ADDITIVE SCHWARZ METHOD FOR VARIATIONAL INEQUALITIES
}

\author{
LORI BADEA AND JUNPING WANG
}

\begin{abstract}
This paper proposes an additive Schwarz method for variational inequalities and their approximations by finite element methods. The Schwarz domain decomposition method is proved to converge with a geometric rate depending on the decomposition of the domain. The result is based on an abstract framework of convergence analysis established for general variational inequalities in Hilbert spaces.
\end{abstract}

\section{INTRODUCTION}

Over the last two decades, there has been a very active study of the domain decomposition method for approximate solutions of partial differential equations. This study was motivated by an increasing need for the solution of large-scale problems in sciences and engineering. The domain decomposition method has the capability of providing numerical solvers which are portable, efficient, and parallelizable on parallel machines.

The problem of variational inequalities arises from many physical and economical applications such as fluid flow in porous media, the behavior of elasto-plastic materials and lubrification phenomena in mechanics, and valuation of American options in modern finance. Those problems are in general of free boundary type and share features similar to the well-known obstacle problem. The method of variational inequalities is a powerful technique to tackle such problems in theory, and also in practical computation.

The multiplicative and additive Schwarz methods for elliptic linear problems have been studied by many researchers, among them Lions [14]-16], Chan, Hou and Lions [4, Bramble, Pasciak, Wang and Xu [3], and Badea [1], for the multiplicative methods (the classical Schwarz alternating algorithm), and Dryja [5], Dryja and Widlund [6], 7] and Nepomnyashchikh [17, for the additive version. For problems related to variational inequalities, there are results by Lions [14, Hoffman and Zou 8], Kuznetsov and Neittaanmäki [10, Kuznetsov, Neittaanmäki and Tarvainen [1]-12, Lü, Liem and Shih [18, Tarvainen [19, Badea 2], and Zeng and Zhou [20] in the domain decomposition method, and by Kornhuber [9] and Mandel [13] in the multigrid method. Despite those results, the functioning and convergence of

Received by the editor December 16, 1997 and, in revised form, September 22, 1998.

1991 Mathematics Subject Classification. 65K10, 65J99, 35R35, 35J60, 49D27, 49D37.

Key words and phrases. variational inequalities, obstacle problems, finite element methods, domain decomposition methods.

The research of Wang is supported in part by National Science Foundation Grant \# DMS9706985 .

(C)2000 American Mathematical Society 
the Schwarz domain decomposition method is not fully understood for variational inequalities.

The objective of this paper is to exploit a convergence theory for the additive Schwarz method. As revealed in [14, 3, 6] for elliptic equations, a good convergence often results from norm estimates for some error reduction operators. While the existence of such error reduction operators is still a question in many applications, the corresponding norm estimate is non-trivial, due to the fact that the operators involved are related to projections onto closed and convex subsets of a Hilbert space.

We shall reformulate the additive Schwarz algorithm in a way which admits a nice recurrence for the errors between two consecutive steps. Through a study for projection operators onto closed and convex subsets of a Hilbert space, we shall prove a geometric convergence for the additive Schwarz method. For simplicity, the theory will be demonstrated for the obstacle problem only.

The paper is organized as follows. In Section 2, we study the variational inequality in an abstract framework. The general result developed in Section 2 shall be applied to an obstacle problem in Sobolev space in Section 3. In Section 4 we analyze the Schwarz method in the finite element space.

\section{An ABStract FramewORK}

2.1. An iterative scheme. Let $V$ be a real Hilbert space with inner product $(\cdot, \cdot)$, and $a(\cdot, \cdot)$ a symmetric bilinear form, defined on $V \times V$, which is bounded and coercive. Let $K$ be a closed convex subset of $V$. Consider the abstract problem which seeks $u \in K$ satisfying

$$
a(u, v-u) \geq f(v-u), \quad \forall v \in K,
$$

where $f$ is a bounded linear functional on $V$. Since the bilinear form $a(\cdot, \cdot)$ is equivalent to the original inner product of the Hilbert space $V$, we may identify $a(\cdot, \cdot)$ as the inner product of $V$.

The problem (2.1) is equivalent to the following minimization problem: Find $u \in K$ such that

$$
F(u) \leq F(v), \quad \forall v \in K,
$$

where $F(v)=\frac{1}{2} a(v, v)-f(v)$ is a functional defined on the Hilbert space $V$.

We would like to approximate the solution of (2.1) by iterative procedures. To this end, let $V_{i}, i=1, \cdots, J$, be a sequence of subspaces of $V$ such that

$$
V=V_{1}+V_{2}+\cdots+V_{J}
$$

Let $u_{n} \in K$ be the current approximation of the exact solution $u$ of (2.1). We correct $u_{n}$ in the subspace $V_{i}$ by seeking $v_{i} \in V_{i}$ such that $u_{n, i}=u_{n}+v_{i} \in K$ and

$$
F\left(u_{n}+v_{i}\right) \leq F\left(u_{n}+v\right), \quad \forall v \in V_{i}, u_{n}+v \in K .
$$

By introducing a convex set

$$
K_{n, i}=\left\{v: \quad v \in V_{i}, v+u_{n} \in K\right\}
$$

we can make the above problem equivalent to the seeking of $v_{i} \in K_{n, i}$ satisfying

$$
F\left(u_{n}+v_{i}\right) \leq F\left(u_{n}+v\right), \quad \forall v \in K_{n, i} .
$$


The solution $v_{i}$ of (2.3) is considered as a correction of $u_{n}$ in $V_{i}$. The total correction for $u_{n}$ is $\sum_{i} v_{i}$, which, in general, over-corrects $u_{n}$. Therefore, we shall scale the total correction by a scalar $\rho>0$ and update $u_{n}$ by

$$
u_{n+1}=u_{n}+\rho \sum_{i=1}^{J} v_{i} .
$$

The parameter $\rho$ in (2.4) should be selected such that $u_{n}+\rho \sum_{i=1}^{J} v_{i} \in K$. In particular, any $\rho \leq 1 / J$ would be a good candidate, since, with $\mu=\rho J \leq 1$, we have

$$
\begin{aligned}
u_{n}+\rho \sum_{i=1}^{J} v_{i} & =(1-\mu) u_{n}+\mu\left(u_{n}+\frac{1}{J} \sum_{i=1}^{J} v_{i}\right) \\
& =(1-\mu) u_{n}+\mu \sum_{i=1}^{J} \frac{1}{J}\left(u_{n}+v_{i}\right),
\end{aligned}
$$

which is a convex sum of vectors in the convex set $K$.

The minimization problem (2.3) can be written as a variational inequality which seeks $v_{i} \in K_{n, i}$ such that

$$
a\left(u_{n}+v_{i}, v-v_{i}\right) \geq f\left(v-v_{i}\right), \quad \forall v \in K_{n, i} .
$$

For standard equalities, one can replace $f\left(v-v_{i}\right)$ in (2.5) by $a\left(u, v-v_{i}\right)$, with $u$ being the exact solution of the original problem. This substitution is generally prohibited for variational inequalities, due to the nonlinearity of the problem. For sake of convergence analysis, we shall make the following assumption:

H1: The problem (2.5) is equivalent to finding $v_{i} \in K_{n, i}$ such that

$$
a\left(u_{n}+v_{i}, v-v_{i}\right) \geq a\left(u, v-v_{i}\right), \quad \forall v \in K_{n, i} .
$$

In other words, the correction is given by solving

$$
a\left(v_{i}, v-v_{i}\right) \geq a\left(u-u_{n}, v-v_{i}\right), \quad \forall v \in K_{n, i} .
$$

If $P_{n, i}$ denotes the projection operator onto the closed convex subset $K_{n, i}$, then (2.6) says that the correction $v_{i}$ of $u_{n}$ in the subspace $V_{i}$ is the projection of the difference $u-u_{n}$ in $K_{n, i}$. Thus,

$$
v_{i}=P_{n, i}\left(u-u_{n}\right) .
$$

The following is a compact form of the proposed iterative scheme.

Algorithm 2.1. Starting from any $u_{0} \in K$, one computes a sequence of approximations $\left\{u_{n}\right\}$ as follows:

1. Compute the projection of the error $u-u_{n}$ on $K_{n, i}$ by solving $v_{i} \in K_{n, i}$ from (2.7) or (2.5).

2. Compute the new approximation $u_{n+1}$ from (2.4).

Let $e_{n}=u-u_{n}$ be the error at step $n$. Since $u_{n+1}$ is given by (2.4) and $v_{i}=P_{n, i}\left(u-u_{n}\right)$, then

$$
u_{n+1}=u_{n}+\rho \sum_{i=1}^{J} P_{n, i}\left(u-u_{n}\right) .
$$

It follows that

$$
u-u_{n+1}=u-u_{n}-\rho \sum_{i=1}^{J} P_{n, i}\left(u-u_{n}\right) .
$$


This can be rewritten as

$$
e_{n+1}=\left(I-\rho \sum_{i=1}^{J} P_{n, i}\right) e_{n} .
$$

By introducing an additive operator

$$
T_{n}=\sum_{i=1}^{J} P_{n, i}
$$

we arrive at the following error recurrence:

$$
e_{n+1}=\left(I-\rho T_{n}\right) e_{n},
$$

which resembles the error reduction relation for equalities. Thus, convergence of the Schwarz method can be established by analyzing the additive operator $T_{n}$.

2.2. Some technical estimates. We present some inequalities valuable for studying the convergence of the abstract iterative Algorithm 2.1. For simplicity, we assume that $a(\cdot, \cdot) \equiv(\cdot, \cdot)$.

Let $K_{i}, i=1, \cdots, J$, be a set of convex and closed subsets of $V$. Denote by $P_{i}$ the projection operator onto $K_{i}$. Recall that the projection $P_{i} w \in K_{i}$ for any $w \in V$ is defined as the solution of the following inequality:

$$
\left(P_{i} w, v-P_{i} w\right) \geq\left(w, v-P_{i} w\right), \quad \forall v \in K_{i} .
$$

The corresponding additive operator is given by

$$
T=P_{1}+P_{2}+\cdots+P_{J}
$$

Assume that each convex set $K_{i}$ contains the vector zero. This assumption is satisfied for $K_{i}=K_{n, i}$ since, by assumption, $u_{n}$ is a vector in $K$. By taking $v=0$ in (2.10), one has the following:

$$
\left(P_{i} w, P_{i} w\right) \leq\left(w, P_{i} w\right), \quad \forall w \in V .
$$

Furthermore, (2.10) implies

$$
\left(w, v-P_{i} w\right) \leq\left(P_{i} w, v-P_{i} w\right) \leq\left(P_{i} w, v\right), \quad \forall v \in K_{i} .
$$

Definition 2.1. A vector $w \in V$ is said to have an admissible decomposition with respect to $\left\{K_{i}\right\}$ and a fixed constant $C_{0}$ if there exists a partition of $w$,

$$
w=w_{1}+w_{2}+\cdots+w_{J}, \quad w_{i} \in K_{i},
$$

such that

$$
\sum_{i=1}^{J}\left\|w_{i}\right\|^{2} \leq C_{0}\|w\|^{2}
$$

Lemma 2.1. If $w$ has an admissible decomposition with respect to $\left\{K_{i}\right\}$ and the constant $C_{0}$, then

$$
(w, w) \leq\left(2+C_{0}\right)(w, T w) .
$$

Proof. Using (2.14), one has

$$
(w, w)=\sum_{i=1}^{J}\left(w, w_{i}\right)=\sum_{i=1}^{J}\left(w, w_{i}-P_{i} w\right)+\sum_{i=1}^{J}\left(w, P_{i} w\right) .
$$


From (2.13), we have $\left(w, w_{i}-P_{i} w\right) \leq\left(P_{i} w, w_{i}\right)$. Thus,

$$
(w, w) \leq \sum_{i=1}^{J}\left(P_{i} w, w_{i}\right)+\sum_{i=1}^{J}\left(w, P_{i} w\right) .
$$

Apply the Schwarz inequality to the first term on the right-hand side of (2.18):

$$
\sum_{i=1}^{J}\left(P_{i} w, w_{i}\right) \leq \sum_{i=1}^{J}\left\|P_{i} w\right\|\left\|w_{i}\right\| \leq\left(\sum_{i=1}^{J}\left\|P_{i} w\right\|^{2}\right)^{1 / 2}\left(\sum_{i=1}^{J}\left\|w_{i}\right\|^{2}\right)^{1 / 2} .
$$

Using the assumption (2.15) and (2.12), we arrive at

$$
\sum_{i=1}^{J}\left(P_{i} w, w_{i}\right) \leq \sqrt{C_{0}}\|w\|\left(\sum_{i=1}^{J}\left(w, P_{i} w\right)\right)^{1 / 2} \leq \frac{1}{2}\|w\|^{2}+\frac{C_{0}}{2} \sum_{i=1}^{J}\left(w, P_{i} w\right) .
$$

Substituting the above into (2.18) gives

$$
\|w\|^{2} \leq \frac{1}{2}\|w\|^{2}+\left(1+C_{0} / 2\right) \sum_{i=1}^{J}\left(w, P_{i} w\right),
$$

which can be rewritten as

$$
\|w\|^{2} \leq\left(2+C_{0}\right) \sum_{i=1}^{J}\left(w, P_{i} w\right)=\left(2+C_{0}\right)(w, T w) .
$$

This completes the proof of the lemma.

Next, we investigate the boundedness of the operator $T$. To this end, assume that $\tau_{i j} \in[0,1]$ is a real number that satisfies

$$
\left|\left(P_{i} w, P_{j} v\right)\right| \leq \tau_{i j}\left\|P_{i} w\right\|\left\|P_{j} v\right\| \quad \forall w, v \in V .
$$

Let $\tau=\left(\tau_{i j}\right)_{J \times J}$, and let $|\tau|$ be the norm of the matrix $\tau$ with respect to the $\ell^{2}$ norm in $\mathbf{R}^{J}$.

Lemma 2.2. Let $T$ be defined as above. Then,

$$
\|T w\|^{2} \leq|\tau|(w, T w), \quad \forall w \in V .
$$

Consequently,

$$
\|T w\|^{2} \leq|\tau|^{2}\|w\|^{2}, \quad \forall w \in V .
$$

Proof. From (2.11),

$$
\|T w\|^{2}=(T w, T w)=\sum_{i, j=1}^{J}\left(P_{i} w, P_{j} w\right) .
$$

Using the inequality (2.19), we obtain

$$
\|T w\|^{2} \leq \sum_{i, j=1}^{J} \tau_{i j}\left\|P_{i} w\right\|\left\|P_{j} w\right\| \leq|\tau| \sum_{i=1}^{J}\left\|P_{i} w\right\|^{2} .
$$

Thus, it follows from (2.12) that

$$
\|T w\|^{2} \leq|\tau| \sum_{i=1}^{J}\left(w, P_{i} w\right)=|\tau|(w, T w),
$$


which verifies (2.20). The proof of (2.21) is a direct application of (2.20) and the Cauchy-Schwarz inequality.

The result on the operator $T$ is summarized as follows:

Theorem 2.1. If $w \in V$ has an admissible decomposition with respect to $\left\{K_{i}\right\}$ and the constant $C_{0}$, then

$$
\left(2+C_{0}\right)^{-1}\|w\|^{2} \leq(w, T w) \leq|\tau|\|w\|^{2},
$$

and

$$
\left(2+C_{0}\right)^{-2}\|w\|^{2} \leq\|T w\|^{2} \leq|\tau|^{2}\|w\|^{2} .
$$

Proof. The left part of (2.22) was seen in Lemma 2.1 The right part is proved by using Lemma 2.2 as follows:

$$
(w, T w) \leq\|w\|\|T w\| \leq|\tau|\|w\|^{2} .
$$

The right part of (2.23) has been proved by Lemma 2.2. The left part can be verified by using Lemma 2.1 as follows:

$$
\|w\|^{2} \leq\left(2+C_{0}\right)(w, T w) \leq\left(2+C_{0}\right)\|w\|\|T w\|,
$$

which leads to

$$
\|w\|^{2} \leq\left(2+C_{0}\right)^{2}\|T w\|^{2}
$$

2.3. Convergence. Let us now investigate the convergence of the iterative Algorithm 2.1.

Theorem 2.2. Let $u_{n+1}$ be given by (2.4), and let $u$ be the solution of (2.1). Assume that the assumption $\mathbf{H 1}$ is satisfied. Assume that the initial guess $u_{0} \in K$ and that, at each iterative step $n, u-u_{n}$ has an admissible decomposition with respect to $\left\{K_{n, i}\right\}$ and a fixed constant $C_{0}$ independent of $n$. Then, there exists a fixed $\tau_{0} \in(0,1)$ such that

$$
\left\|u-u_{n+1}\right\|^{2} \leq \tau_{0}\left\|u-u_{n}\right\|^{2}
$$

provided that the parameter $\rho$ is sufficiently small.

Proof. The error recurrence for $\left\{u_{n}\right\}$ is given by (2.9):

$$
e_{n+1}=\left(I-\rho T_{n}\right) e_{n} .
$$

It follows that

$$
\left\|e_{n+1}\right\|^{2}=\left\|e_{n}\right\|^{2}-2 \rho\left(T_{n} e_{n}, e_{n}\right)+\rho^{2}\left(T_{n} e_{n}, T_{n} e_{n}\right) .
$$

Using Theorem 2.1 we obtain

$$
\left\|e_{n+1}\right\|^{2} \leq\left(1-2 \rho\left(2+C_{0}\right)^{-1}+\rho^{2}|\tau|^{2}\right)\left\|e_{n}\right\|^{2} .
$$

Thus, there exists an $\tau_{0} \in(0,1)$ such that

$$
1-2 \rho\left(2+C_{0}\right)^{-1}+\rho^{2}|\tau|^{2} \leq \tau_{0}
$$

for sufficiently small $\rho$. This proves our theorem. 
Remark 2.1. The function

$$
g(\rho)=1-2 \rho\left(2+C_{0}\right)^{-1}+\rho^{2}|\tau|^{2}
$$

attains its minimum value

$$
g\left(\rho_{0}\right)=1-\frac{1}{\left(2+C_{0}\right)|\tau|^{2}}
$$

at $\rho_{0}=1 /\left(2+C_{0}\right)|\tau|^{2}$. But such a $\rho_{0}$ may not be a candidate, since the corresponding approximation $u_{n+1}=u_{n}+\rho_{0} T_{n}\left(u-u_{n}\right)$ may not be a vector in $K$.

\section{An APplication in the DOMAin DECOMPOSItion MEthod}

The abstract result established in Section 2 has applications in many applied sciences such as fluid flow in porous media, obstacle problems, and the valuation of American options in modern finance. For simplicity, we shall illustrate the idea for obstacle problems only.

Let $\Omega$ be an open bounded domain in $\mathbf{R}^{n}, n \in \mathbf{N}$, with Lipschitz continuous boundary $\Gamma=\partial \Omega$. Assume that $\partial \Omega=\bar{\Gamma}_{1} \cup \bar{\Gamma}_{2}, \Gamma_{1} \cap \Gamma_{2}=\emptyset$, is a partition of the boundary such that meas $\left(\Gamma_{1}\right)>0$. We consider the Sobolev space $V=\{v \in$ $H^{1}(\Omega): v=0$ on $\left.\Gamma_{1}\right\}$, the convex set

$$
K=\{v \in V: v \geq 0 \text { in } \Omega\},
$$

and the problem of finding $u \in K$ such that

$$
a(u, v-u) \geq f(v-u), \quad \forall v \in K,
$$

where $a(\cdot, \cdot)$ is a symmetric, continuous, and positive definite bilinear form on $V \times V$ and $f \in V^{\prime}, V^{\prime}$ being the dual of $V$.

We remark that if the convex set $K$ of the above problem is of the form $\{v \in$ $V: v \geq \alpha$ in $\Omega\}, \alpha \in V$, or $\{v \in V: v \leq \beta$ in $\Omega\}, \beta \in V$, by taking $w=u-\alpha$ or $w=\beta-u$ we can reduce the problem to a study of the inequality (3.2) with a convex set given as in (3.1).

For simplicity, the analysis can be restricted to the following model bilinear form:

$$
a(v, w)=\int_{\Omega} \nabla v \cdot \nabla w d x, \quad v, w \in V .
$$

Observe that the problem (3.2) has a unique solution in the given convex set $K$. Our objective is to approximate the solution $u$ efficiently by using an additive Schwarz domain decomposition method.

First, we decompose the domain into overlapping subdomains:

$$
\Omega=\bigcup_{i=1}^{J} \Omega_{i},
$$

where the $\Omega_{i}$ 's are open subdomains with Lipschitz continuous boundary. Second, we define

$$
V_{i}=\left\{v_{i} \in V: \quad v=0 \text { in } \Omega-\bar{\Omega}_{i}\right\},
$$

for $i=1, \cdots, J$. Next, we apply the abstract theory of Section 2 to approximate the solution $u$ of (3.2). The following is a slightly different but equivalent statement of the iterative Algorithm [2.1.

Algorithm 3.1. Starting from any initial guess $u_{0} \in K$, the method computes a sequence of approximations $\left\{u_{n}\right\}$ as follows: 
1. Assume that $u_{n}$ is known. Consider the convex set

$$
K_{n, i}=\left\{v_{i} \in V_{i}: v_{i}+u_{n} \in K\right\} .
$$

For each $i \in\{1, \cdots, J\}$, compute $v_{n, i} \in K_{n, i}$ by solving

$$
a\left(u_{n}+v_{n, i}, v-v_{n, i}\right) \geq f\left(v-v_{n, i}\right), \quad \forall v \in K_{n, i} .
$$

2. Update the approximation by

$$
u_{n+1}=u_{n}+\rho \sum_{i=1}^{J} v_{n, i},
$$

where $\rho$ should be selected so that $u_{n+1} \in K$.

The parameter $\rho$ is not necessarily a constant in the Schwarz method. For example, one can use any smooth positive function $\rho=\rho(x)$ in (3.7). The following lemma provides a useful criterion for the selection of $\rho$.

Lemma 3.1. For any $x \in \Omega$, let $N(x)$ be the number of subdomains containing $x$. If $\rho$ is selected as a smooth positive function such that

$$
\rho(x) N(x) \leq 1, \quad \forall x \in \Omega,
$$

then the approximation $u_{n+1}$ from (3.7) is a function in the convex set $K$. Also, the convergence proved in Theorem 2.2 holds in this case if

$$
1-2 \rho_{1}\left(1-C_{0}\right)^{-1}+\rho_{2}^{2}|\tau|^{2} \leq \tau_{0}<1,
$$

where $\rho_{1}=\min _{x} \rho(x)$ and $\rho_{2}=\max _{x} \rho(x)$.

Proof. First, for any $x \in \Omega$,

$$
\sum_{i=1}^{J} v_{n, i}(x) \geq N(x) \min _{i} v_{n, i}(x) .
$$

Thus, from (3.7) and (3.8),

$$
\begin{aligned}
u_{n+1}(x) & =u_{n}(x)+\rho(x) \sum_{i=1}^{J} v_{n, i}(x) \\
& \geq u_{n}(x)+\rho(x) N(x) \min _{i} v_{n, i}(x) .
\end{aligned}
$$

If $\min _{i} v_{n, i}(x) \geq 0$, then we naturally have $u_{n+1}(x) \geq 0$ from the above inequality. If $\min _{i} v_{n, i}(x)<0$, then by (3.8)

$$
u_{n+1}(x) \geq u_{n}(x)+\rho(x) N(x) \min _{i} v_{n, i}(x) \geq u_{n}+\min _{i} v_{n, i}(x) \geq 0,
$$

where, at the last step, we have used the fact that $u_{n}(x)+v_{n, i}(x) \geq 0$ for any $i \in J$. Regarding the second part of the lemma, we see that it follows immediately from

$$
\left\|e_{n+1}\right\|^{2} \leq\left(1-2 \rho_{1}\left(2+C_{0}\right)^{-1}+\rho_{2}^{2}|\tau|^{2}\right)\left\|e_{n}\right\|^{2}
$$

instead of 2.25).

The above iterative algorithm is essentially an additive method which can be easily parallelized on parallel machines. To prove convergence by using the abstract result established in Section 2 we ought to show first that the assumption $\mathbf{H 1}$ is 
satisfied for the model problem (3.2). With $u_{n, i}=u_{n}+v_{n, i}$, we can rewrite (2.6) as follows: Find $u_{n, i} \in K_{n, i}+u_{n}$ such that

$$
a\left(u_{n, i}, v-u_{n, i}\right) \geq a\left(u, v-u_{n, i}\right), \quad \forall v \in u_{n}+K_{n, i} .
$$

Then, the assumption $\mathbf{H 1}$ is equivalent to the equivalence of (3.9) and (3.6).

Lemma 3.2. Let $u$ be the solution of (3.2) and $u_{n, i}$ the solution of the inequality (3.9). If the $n$-th step approximation $u_{n}$ satisfies

$$
u_{n} \in K, \quad u-u_{n} \in K,
$$

then

$$
u_{n, i} \in K, \quad u-u_{n, i} \in K
$$

Furthermore, the inequalities (3.9) and (3.6) are equivalent in the sense that if $u_{n, i}$ solves (3.9) and $v_{n, i}$ solves (3.6), then $u_{n, i}=u_{n}+v_{n, i}$.

Proof. 1. Let

$$
\Omega_{i}^{+}=\left\{x \in \Omega_{i}: u_{n, i}>0\right\} .
$$

By letting $v=u_{n, i} \pm \epsilon w_{i}$ in (3.9) we get

$$
a\left(u_{n, i}-u, w_{i}\right)=0, \quad \forall w_{i} \in V_{i}, w_{i}=0 \text { on } \Omega-\bar{\Omega}_{i}^{+} .
$$

2. We show that $u-u_{n, i} \in K$. To this end, let

$$
D_{i}=\left\{x \in \Omega: u_{n, i}-u>0\right\} .
$$

We claim that $D_{i} \subset \Omega_{i}^{+}$. In fact, if $x \in D_{i}$, then

$$
u_{n, i}(x)>u(x) \geq 0 \text {. }
$$

Since in $\Omega-\Omega_{i}$ we have $u_{n, i}=u_{n} \leq u$, then (3.11) implies that $x \in \Omega_{i}$ and $u_{n, i}(x)>0$. Hence, $x \in \Omega_{i}^{+}$.

Observe that $u-u_{n} \in K$ (i.e., $u \geq u_{n}$ ) and, therefore, $u_{n, i}-u=u_{n}-u \leq 0$ on $\partial \Omega_{i} \cap \Omega$. Since $D_{i} \subset \Omega_{i}^{+}$, the function

$$
\phi_{i}= \begin{cases}u_{n, i}(x)-u(x), & x \in D_{i}, \\ 0, & x \in \Omega-D_{i},\end{cases}
$$

lies in $V_{i}$ and vanishes in $\Omega-\Omega_{i}^{+}$. Replacing $w_{i}$ in (3.10) by $\phi_{i}$ gives

$$
a\left(\phi_{i}, \phi_{i}\right)=0 \text {, }
$$

which implies that $\phi_{i} \equiv 0$. Thus, $D_{i}$ must be the empty set. This shows that $u-u_{n, i} \geq 0$ and, therefore, $u-u_{n, i} \in K$.

3. Now we show that the inequalities (3.9) and (3.6) are equivalent in the sense stated in the theorem. In fact, from (3.9) we have

$$
a\left(u_{n, i}, v-u_{n, i}\right) \geq a\left(u, v-u_{n, i}\right)=a(u, v)-a\left(u, u_{n, i}\right),
$$

for any $v \in K_{n, i}+u_{n} \subset K$. It is clear that

$$
a(u, v) \geq f(v),
$$

and since $u-u_{n, i} \in K$, we have

$$
-a\left(u, u_{n, i}\right)=a\left(u, u-u_{n, i}-u\right) \geq f\left(u-u_{n, i}-u\right)=f\left(-u_{n, i}\right) .
$$

Substituting the last two relations into (3.12), we obtain

$$
a\left(u_{n, i}, v-u_{n, i}\right) \geq f\left(v-u_{n, i}\right), \quad \forall v \in u_{n}+K_{n, i} .
$$


By letting $v_{n, i}=u_{n, i}-u_{n}$, we see that $v_{n, i}$ provides a solution for (3.6). This, together with the uniqueness of the solution of (3.9) and (3.6), yields the desired equivalence.

We have already proved in Lemma 3.1 that the new approximation $u_{n+1}$ lies in $K$ as long as $u_{n} \in K$ and $\rho(x) N(x) \leq 1$. Assume that $u-u_{n} \in K$. We would like to know if $u-u_{n+1} \in K$ is valid under the same constraint on $\rho$. The answer is positive. To see why, first observe that, by (3.7),

$$
u-u_{n+1}=u-u_{n}-\rho \sum_{i=1}^{J} v_{n, i} .
$$

Notice that from Lemma 3.2 we have $u_{n}+v_{n, i}=u_{n, i} \leq u$. Thus,

$$
\sum_{i=1}^{J} v_{n, i}(x) \leq N(x)\left(u(x)-u_{n}(x)\right) \text {. }
$$

It follows that

$$
u-u_{n}-\rho \sum_{i=1}^{J} v_{n, i} \geq u-u_{n}-\rho N(x)\left(u-u_{n}\right)=(1-\rho N)\left(u-u_{n}\right) \geq 0 .
$$

Hence, $u-u_{n+1} \in K$. The result can be summarized as follows:

Theorem 3.1. Let $u$ be the solution of the inequality (3.2), and let $\left\{u_{n}\right\}$ be a sequence of approximations given by Algorithm 3.1 in which the parameter $\rho$ is selected according to Lemma 3.1. If the initial guess $u_{0}$ is selected such that $u_{0}, u-$ $u_{0} \in K$, then $u_{n+1}, u-u_{n+1} \in K$. Furthermore, the problem (3.6) is equivalent to (3.9) in the sense that $u_{n, i}=u_{n}+v_{n, i}$.

Let us investigate the convergence of the proposed iterative Algorithm 3.1 Since the method fits the general framework proposed in Section 2, we can obtain convergence by verifying the conditions of Theorem 2.2.

First, we claim that the matrix $\tau$ is bounded. In fact, the boundedness of $\tau$ depends on the number of overlaps (namely, the function $N(x)$ ) in the domain decomposition. In practical computations, the domain $\Omega$ is often decomposed so that $|\tau|$ has an upper bound independent of the number of subdomains.

Second, we claim that, under certain conditions imposed on the domain decomposition, at each iterative step $n$, the error $u-u_{n}$ has an admissible decomposition with respect to $\left\{K_{n, i}\right\}$ and a certain constant $C_{0}$. In fact, for a certain decomposition of the domain (see [1] or [14, for instance) we can find some sufficiently smooth functions $\left\{\phi_{i}\right\}$ with $\operatorname{supp}\left(\phi_{i}\right) \subset \bar{\Omega}_{i}$ satisfying in $\Omega$

$$
0 \leq \phi_{i} \leq 1, \quad \sum_{i=1}^{J} \phi_{i} \equiv 1 .
$$

With $v_{i}=\left(u-u_{n}\right) \phi_{i}$, we have

$$
u-u_{n}=\sum_{i=1}^{J} v_{i}
$$

and $v_{i} \in K_{n, i}$. Furthermore, there exists a constant $C_{0}$, depending only on $\left\{\phi_{i}\right\}$, such that (2.15) is satisfied. 


\section{An APPLiCATION TO Finite ELEMENT APPROXIMATIONS}

Consider the approximate solution of (B.2) by finite element methods. Let $V_{h}$ be a finite element subspace of $V$ and denote by

$$
K_{h}=\left\{v_{h} \in V_{h}: v_{h}(x) \geq 0 \forall \text { nodal points } x\right\}
$$

a convex subset of $V_{h}$. The discrete problem of (3.2) seeks $u_{h} \in K_{h}$ such that

$$
a\left(u_{h}, v-u_{h}\right) \geq f\left(v-u_{h}\right), \quad \forall v \in K_{h} .
$$

Observe that $K_{h}$ need not be a subset of $K$.

Our objective here is to approximate $u_{h}$ by overlapping domain decomposition methods analogous to the method described in the previous section for the continuous problem.

To this end, assume that the domain $\Omega$ is decomposed into a set of overlapping subdomains satisfying (3.4), and that the meshes of the subdomains align well with that of $\Omega$ along the boundary. Corresponding to each subdomain $\Omega_{i}$, we have a subspace $V_{h i}=V_{h} \cap V_{i}$ for $i=1, \cdots, J$.

The following is an iterative algorithm using the information provided by each subdomain. The algorithm resembles Algorithm 3.1 in appearance.

Algorithm 4.1. Starting from any initial guess $u_{0}^{h} \in K_{h}$, the method computes a sequence of approximations $\left\{u_{k}^{h}\right\}$ as follows:

1. Assume that $u_{n}^{h}$ is known. Consider the convex set

$$
K_{n, i}=\left\{w_{i} \in V_{h i}: w_{i}+u_{n}^{h} \in K_{h}\right\} .
$$

For each $i \in\{1, \cdots, J\}$, compute $v_{i} \in K_{n, i}$ by solving

$$
a\left(u_{n}^{h}+v_{i}, v-v_{i}\right) \geq f\left(v-v_{i}\right), \quad \forall v \in K_{n, i} .
$$

2. Update the approximation by

$$
u_{n+1}^{h}=u_{n}^{h}+\rho \sum_{i=1}^{J} v_{i}
$$

The convergence of Algorithm 4.1 can be established by using the general result presented in Section 2. The analysis is similar to the one in Section 3 for the continuous case. The only difficulty here is in the verification of the assumption H1. Observe that this assumption really requires the equivalence of (4.3) with the following problem:

$$
a\left(u_{n}^{h}+v_{n, i}, v-v_{n, i}\right) \geq a\left(u_{h}, v-v_{n, i}\right), \quad \forall v \in K_{n, i} .
$$

In the following, we shall use the notation $u_{n, i}^{h}=u_{n}^{h}+v_{n, i}$.

Lemma 4.1. Let $\left\{\varphi_{i}\right\}_{i=1, \cdots, N}$ be the standard nodal basis function of the finite element space $V_{h}$. Assume that

$$
a\left(\varphi_{j}, \varphi_{k}\right) \leq 0 \text { whenever } j \neq k .
$$

If the iterative approximation $u_{n}^{h}$ satisfies

$$
u_{n}^{h} \in K_{h}, \quad u_{h}-u_{n}^{h} \in K_{h},
$$

then

$$
u_{n, i}^{h} \in K_{h}, \quad u_{h}-u_{n, i}^{h} \in K_{h} .
$$


Proof. It is not hard to see that $u_{n, i}^{h}$ is also characterized as the solution of

$$
a\left(u_{n, i}^{h}, v-u_{n, i}^{h}\right) \geq a\left(u_{h}, v-u_{n, i}^{h}\right), \quad \forall v \in K_{n, i}+u_{n}^{h} .
$$

1. Let $\mathcal{N}=\left\{x_{j}\right\}$ be the set of nodal points. Denote by $\mathcal{N}_{n, i}^{+}$the subset of $\mathcal{N}$ on which $u_{n, i}^{h}>0$. It is routine to show that

$$
a\left(u_{n, i}^{h}-u_{h}, \varphi_{j}\right)=0
$$

for any nodal basis $\varphi_{j}$ corresponding to nodal points in $\mathcal{N}_{n, i}^{+}$.

2. Let us verify that $u_{h}-u_{n, i}^{h} \in K_{h}$. Observe that it is equivalent to

$$
u_{h}\left(x_{j}\right)-u_{n, i}^{h}\left(x_{j}\right) \geq 0, \quad \forall x_{j} \in \mathcal{N} .
$$

Denote by $\mathcal{M}_{i}$ the subset of $\mathcal{N}$ on which

$$
u_{n, i}^{h}\left(x_{j}\right)-u_{h}\left(x_{j}\right)>0, \quad x_{j} \in \mathcal{M}_{i} .
$$

We wish to show that $\mathcal{M}_{i}=\emptyset$. To this end, we consider the following decomposition:

$$
u_{n, i}^{h}(x)-u_{h}(x)=\sum_{x_{j} \in \mathcal{M}_{i}} \varrho_{j} \varphi_{j}(x)+\sum_{x_{k} \in \mathcal{N}-\mathcal{M}_{i}} \varrho_{k} \varphi_{k}(x),
$$

where $\varrho_{j}=u_{n, i}^{h}\left(x_{j}\right)-u_{h}\left(x_{j}\right)$. Call the first term on the right-hand side of (4.11) $w_{1}$ and the second term $w_{2}$. Observe that (4.10) implies $\mathcal{M}_{i} \subset \mathcal{N}_{n, i}^{+}$. It follows from (4.8) that

$$
a\left(u_{n, i}^{h}-u_{h}, w_{1}\right)=0
$$

which leads from (4.11) to

$$
a\left(w_{1}+w_{2}, w_{1}\right)=0 .
$$

Thus,

$$
a\left(w_{1}, w_{1}\right)=-a\left(w_{2}, w_{1}\right)=-\sum_{j, k} \varrho_{j} \varrho_{k} a\left(\varphi_{k}, \varphi_{j}\right) .
$$

Notice that $\varrho_{j}>0, \varrho_{k} \leq 0$, and $a\left(\varphi_{k}, \varphi_{j}\right) \leq 0$. Thus, the right-hand side of (4.12) is non-positive. This together with the coercivity of the bilinear form $a(\cdot, \cdot)$ shows that $w_{1}=0$, which is possible only if $\mathcal{M}_{i}=\emptyset$.

Similarly to the proof of Theorem 3.1, one can show that the sequence of approximations $\left\{u_{n}^{h}\right\}$ satisfies (4.6), provided the initial guess $u_{0}^{h}$ is selected with the same property. The next theorem gives the equivalence of inequalities (4.3) and (4.7), which verifies the validity of the assumption $\mathbf{H} \mathbf{1}$ for the present application.

Theorem 4.1. Assume that (4.5) is satisfied, $u_{h}$ is the solution of (4.1), and the initial guess is selected to satisfy (4.6). Let $\left\{u_{n}^{h}\right\}$ be the sequence of approximations obtained from Algorithm 4.1. Then, each iterative approximation $u_{n}^{h}$ satisfies (4.6). Moreover, the inequalities (4.3) and (4.7) are equivalent in the sense that if $v_{i}$ solves (4.3) and $u_{n, i}^{h}$ solves (4.7), then $v_{i}=u_{n, i}^{h}-u_{n}^{h}$.

Proof. It is clear that (4.3) is equivalent to finding $\tilde{u}_{n, i}^{h}=u_{n}^{h}+v_{i} \in K_{n, i}+u_{n}^{h}$ satisfying

$$
a\left(\tilde{u}_{n, i}^{h}, v-\tilde{u}_{n, i}^{h}\right) \geq f\left(v-\tilde{u}_{n, i}^{h}\right) \quad \forall v \in K_{n, i}+u_{n}^{h} .
$$

It suffices to prove the equivalence of (4.13) with (4.7). 
Let $u_{n, i}^{h}$ be the solution of (4.7). It follows that

$$
\begin{aligned}
a\left(u_{n, i}^{h}, v-u_{n, i}^{h}\right) & \geq a\left(u_{h}, v-u_{n, i}^{h}\right) \\
& =a\left(u_{h}, v\right)-a\left(u_{h}, u_{n, i}^{h}\right) \\
& \geq f(v)+a\left(u_{h}, u_{h}-u_{n, i}^{h}-u_{h}\right),
\end{aligned}
$$

for all $v \in K_{n, i}+u_{n}^{h}$. Since $u_{h}-u_{n, i}^{h} \in K_{h}$, then

$$
a\left(u_{n, i}^{h}, v-u_{n, i}^{h}\right) \geq f\left(v-u_{n, i}^{h}\right),
$$

which shows that the solution of (4.7) also solves (4.13). This completes the equivalence, since both problems have a unique solution.

Finally, we remark that it is always possible to construct an admissible decomposition for $u_{h}-u_{n}^{h}$ with respect to $K_{n, i}$ and a constant $C_{0}$ in the finite element method. Consequently, the general convergence result of Section 2 assures geometrical convergence of Algorithm 4.1

Concerning the condition (4.5), we shall give a simple example for which it is satisfied. Let us consider the bilinear form given in (3.3) using piecewise linear finite elements for a domain $\Omega \subset \mathbf{R}^{n}, n=1,2,3$.

- When $n=1$, the property (4.5) is evidently satisfied.

- When $n=2$, let $T$ be a triangle with vertices $P_{1}, P_{2}$, and $P_{3}$. If $\varphi_{1}$ is the shape function corresponding to $P_{1}$, then the vector $\nabla \varphi_{1}$ is in the same direction as the inner normal vector on the edge $P_{2} P_{3}$. Consequently, the property (4.5) holds true if all the angles of the triangle are acute.

- When $n=3$, let $T$ be a tetrahedron with vertices $P_{1}, P_{2}, P_{3}$, and $P_{4}$. Consider the shape function $\varphi_{1}$ corresponding to $P_{1}$. Again, the gradient vector $\nabla \varphi_{1}$ points in the same direction as the inner normal vector on the triangular face containing $P_{2}, P_{3}$, and $P_{4}$. Thus, if the angle between any two faces is acute, then the property (4.5) holds true.

\section{REFERENCES}

[1] L. Badea, A generalization of the Schwarz alternating method to an arbitrary number of subdomains, Numer. Math., 55 (1989), pp. 61-81. MR 90e:65150

[2] L. Badea, On the Schwarz alternating method with more than two subdomains for nonlinear monotone problems, SIAM J. Numer. Anal., 28 (1991), pp. 179-204. MR 91m:65165

[3] J. Bramble, J. Pasciak, J. Wang and J. Xu, Convergence estimates for product iterative methods with applications to domain decomposition, Math. Comp., 57 (1991), pp. 1-21. MR 92d:65094

[4] T. Chan, T. Hou and P. L. Lions, Geometry related convergence results for domain decomposition algorithms, SIAM J. Numer. Anal., 28 (1991), pp. 378-391. MR 92a:65325

[5] M. Dryja, An additive Schwarz algorithm for two- and three-dimensional finite element elliptic problems, in T. Chan et al., eds., Domain Decomposition Methods, Philadelphia, 1989, SIAM, pp. 168-172. MR 89j:65010

[6] M. Dryja and O. Widlund, Some domain decomposition algorithms for elliptic problems, in L. Hayes and D. Kincaid, eds., Iterative Methods for Large Systems, Boston, 1990, Academic Press, pp. 273-291. MR 91f:65071

[7] M. Dryja and O. Widlund, Towards a unified theory of domain decomposition algorithms for elliptic problems, in T. Chan et al., eds., Third International Symposium on Domain Decomposition Methods for Partial Differential Equations, Philadelphia, 1990, SIAM, pp. 3-21. MR 91m:65294

[8] K. H. Hoffmann and J. Zou, Parallel algorithms of Schwarz variant for variational inequalities, Numer. Funct. Anal. Optim., 13 (1992), pp. 449-462. MR 93k:65050

[9] R. Kornhuber, Monotone multigrid methods for elliptic variational inequalities I, Numer. Math., 69 (1994), pp. 167-184. MR 96d:65201 
[10] Y. Kuznetsov and P. Neittaanmäki, Overlapping domain decomposition methods for the simplified Dirichlet-Signorini problem, in W. Ames and P. van der Houwen, eds., Computational and Applied Mathematics II, Amsterdam, 1992, pp. 297-306. MR 94c:65139

[11] Y. Kuznetsov, P. Neittaanmäki and P. Tarvainen Block relaxation methods for algebraic obstacle problem with M-matrices, East-West J. Numer. Math., 2 (1994), pp. 75-90. MR 95d:65029

[12] Y. Kuznetsov, P. Neittaanmäki and P. Tarvainen Overlapping domain decomposition methods for the obstacle problem, in Y. Kuznetsov et al., eds., Domain Decomposition Methods in Science and Engineering, AMS, Philadelphia, 1994, pp. 271-277. MR 94i:65004

[13] J. Mandel, A multilevel iterative method for symmetric, positive definite linear complementarity problems, Appl. Math. Optimization, 11 (1984), pp. 77-95. MR 85b:90082

[14] P. L. Lions, On the Schwarz alternating method I, in R. Glowinski et al., eds., First International Symposium on Domain Decomposition Methods for Partial Differential Equations, Philadelphia, 1988, SIAM, pp. 2-42. MR 90a:65248

[15] P. L. Lions, On the Schwarz alternating method II, in T. Chan et al., eds., Domain Decomposition Methods, Philadelphia, 1989, SIAM, pp. 47-70. MR 90e:65140

[16] P. L. Lions, On the Schwarz alternating method III, in Chan et al., eds., Third International Symposium on Domain Decomposition Methods for Partial Differential Equations, Philadelphia, 1990, SIAM, pp. 202-223. MR 91g:65226

[17] S. Nepomnyashchikh, Application of domain decomposition to elliptic problems with discontinuous coefficients, in R. Glowinski et al., eds., Fourth International Symposium on Domain Decomposition Methods for Partial Differential Equations, Philadelphia, 1991, SIAM, pp. 242-251. MR 92a:65023

[18] T. Lü, C. Liem and T. Shih, Parallel algorithms for variational inequalities based on domain decomposition, System Sci. Math. Sci., 4 (1991), pp. 341-348. MR 93b:49010

[19] P. Tarvainen, Block relaxation methods for algebraic obstacle problems with M-matrices: theory and applications, Report 63, Univ. of Jyväskylä, Department of Mathematics, Jyväskylä, 1994. MR 97g:90154

[20] J. Zeng and S. Zhou, On monotone and geometric convergence of Schwarz methods for two-sided obstacle problems, SIAM J. Numer. Anal., vol. 35, no. 2, (1998). pp. 600-616. MR 99d:65199

Institute of Mathematics, Romanian Academy of Sciences, Bucharest, Romania

E-mail address: lbadea@stoilow.imar.ro

Department of Mathematics, University of Wyoming, Laramie, Wyoming 82071

E-mail address: junping@uwyo.edu 